\title{
BMJ Open Risk factors for exposure of glaucoma drainage devices: a retrospective observational study
}

\author{
Kelly W Muir, ${ }^{1,2}$ Annie Lim, ${ }^{3}$ Sandra Stinnett, ${ }^{2}$ Anthony Kuo, ${ }^{2}$ Henry Tseng, ${ }^{2}$ \\ Molly M Walsh ${ }^{2}$
}

To cite: Muir KW, Lim A, Stinnett $\mathrm{S}$, et al. Risk factors for exposure of glaucoma drainage devices: a retrospective observational study. BMJ Open 2014;4: e004560. doi:10.1136/ bmjopen-2013-004560

- Prepublication history for this paper is available online. To view these files please visit the journal online (http://dx.doi.org/10.1136/ bmjopen-2013-004560).

Received 29 November 2013 Revised 1 April 2014 Accepted 3 April 2014

CrossMark

\footnotetext{
${ }^{1}$ Durham VA Medical Center, Health Services Research \& Development, Durham, North Carolina, USA

${ }^{2}$ Duke Eye Center, Durham, North Carolina, USA

${ }^{3}$ Kaiser Permanente, Oakland Medical Center, Oakland, California, USA

Correspondence to Dr Kelly W Muir; kelly.muir@duke.edu
}

\begin{abstract}
Objectives: The purpose of this study was to identify risk factors for exposure of glaucoma drainage devices (GDD).

Setting: This retrospective, observational study was conducted in the eye clinic of an academic medical centre.
\end{abstract}

Participants: Participants included 1073 consecutive adults who underwent GDD surgery between 1 January 2005 and 1 January 2011. Participants were included if chart review indicated GDD surgery during the study period and excluded if at least 12 months of clinical follow-up was not available in the medical record.

Primary outcome measure: The primary outcome measure was exposure of the GDD occurring at least 1 month after implant surgery. The characteristics of participants who experienced exposure of the implant were compared to the characteristics of participants who did not experience exposure.

Results: Of the 1073 participants having undergone GDD surgery, 67 experienced exposure of the device. Neither the type of GDD, type of patch graft (eye bank sclera, Tutoplast sclera and Tutoplast pericardium), surgeon, location of GDD, number of GDD previously implanted into the eye, nor history of diabetes or uveitis were associated with likelihood of exposure. Women were more likely than men to experience exposure of the GDD (OR 2.004 (95\% Cl1.170 to $3.431))$ in both univariable $(p=0.011)$ and multivariable $(p=0.013)$ analyses. In survival analysis, exposure of the GDD occurred earlier for women than for men (58 vs 61 months; $p=0.024$ ). White race (vs black) was also associated with increased risk of GDD exposure (OR $1.693(95 \% \mathrm{Cl} 1.011$ to 2.833$))$ in univariable $(p=0.044)$ and multivariable $(p=0.046)$ analyses.

Conclusions: Women are two times more likely to experience GDD exposure than men, independent of age. White race is also a risk factor for exposure.

\section{INTRODUCTION}

The use of glaucoma drainage devices (GDD) to manage glaucoma has increased dramatically over the past two decades. Review of medicare claims data indicates that

\section{Strengths and limitations of this study}

- As a retrospective study, all potentially contributing factors may not have been available for review.

- The study includes a larger number of glaucoma drainage device surgeries with greater variety of devices and patch graft materials than has been reported previously and identifies a gender difference in likelihood of exposure that is a new finding.

the number of trabeculectomy surgeries declined by $>50 \%$ while the number of GDD surgeries increased by $>150 \%$ from 1995 to 2004. ${ }^{1}$ The Tube Versus Trabeculectomy Study has provided evidence that GDD surgery can be at least as effective as trabeculectomy at reducing intraocular pressure and the need for further surgery over a 5 year time frame. ${ }^{2}$ Unfortunately, GDD surgery is not without complications, including erosion of the device through the conjunctiva. Reported rates of GDD exposure in adults range from $3 \%$ to $8 \%$ over the first $1-5$ years following implant surgery. ${ }^{3-7}$ Exposure of the GDD puts the patient at risk for potentially devastating infection ${ }^{8}$; as such, exposure of a GDD warrants surgical revision. Revision of the exposed GDD, however, is challenging. In one large series of revision surgeries for GDD exposure, almost half required additional surgeries following the revision and more than $10 \%$ eventually required removal of the implant. ${ }^{9}$

Previously reported risk factors for GDD exposure include inferior versus superior location of the implant, ${ }^{10} 11$ prior $^{4}$ or concurrent $^{3}$ ocular surgery, use of specific patch graft materials ${ }^{12}{ }^{13}$ and Hispanic race. ${ }^{14}$ Most studies investigating risk factors for exposure include only one type of implant or a limited number of patch graft materials. Based on clinical experience, we hypothesised that 
female gender, older age, white race and total number of GDD in the operative eye may impart an increased risk of GDD exposure. The purpose of this study was to review the longitudinal outcomes of patients having undergone GDD implant surgery including a broad variety GDD implants, patch graft materials and surgeons, with the goal of identifying risk factors for exposure of the GDD.

\section{METHODS}

This study was conducted with approval from the Duke University Institutional Review Board and in compliance with Health Insurance Portability and Accountability Act (HIPAA) regulations; a waiver of informed consent was granted. A retrospective review was conducted of all GDD surgeries performed on patients at least 18 years of age at the Duke Eye Center between 1 January 2005 and 1 January 2011. The Duke Data Unified Content Explorer, $^{15}$ a guided query tool for the Duke Enterprise data warehouse, was utilised to identify all surgeries billed for CPT code 66180 (aqueous shunt to extraocular reservoir). Acknowledging that multiple episodes of GDD exposure in the same patient are unlikely to represent independent events, the dataset was further limited to include only one eye and one GDD surgery for each participant. That is to say, the analysis was conducted at the patient level. Likewise, if a patient underwent multiple surgeries within the study period, only the first GDD surgery performed during the study period was included. In order to optimise capture of GDD exposure events, the dataset was limited to participants with at least 12 months of clinical follow-up. A single chart abstractor (AL) reviewed the medical record for each participant having undergone surgery, noting demographic information such as age, gender and race; details of the operation including type and location of GDD implanted, type of patch graft used and surgeon; and ophthalmic history including history of previous surgery. A random sample of $10 \%$ of the charts was reviewed by a second chart abstractor (KWM) and no differences were noted.

The primary outcome for this investigation was GDD exposure. Exposure of the GDD was defined as clinical recognition of exposure of any part of the device occurring more than 1 month following surgery and requiring repair. We focused on exposure events occurring greater than 1 month after surgery to differentiate exposure from operative wound dehiscence. The number of months between the initial surgery and exposure was noted. Descriptive statistics were derived, including means, medians and SDs. The associations between potential explanatory factors and the outcome of exposure were analysed with logistic regression, testing for interactions when appropriate. ORs and CIs were calculated. Survival analysis was used to compare the time to exposure for specific explanatory variables. We analysed the data using SAS/STAT software (SAS Institute, Inc,
Cary, North Carolina, USA). In all cases, a $p$ value of $<0.05$ was considered statistically significant.

\section{RESULTS}

Between 1 January 2005 and 1 January 2011, 1738 GDD surgeries were performed on 1411 adults at the Duke Eye Center. Excluding cases with less than 12 months of follow-up resulted in 1073 individual GDD surgeries for review. During the study period, 67 of these 1073 GDD implants were noted to have become exposed. The characteristics of the total sample and the cases of exposure are described in the table 1.

Characteristics of participants and association with GDD exposure by univariable analysis.

The duration of follow-up after GDD surgery ranged from 12 to 84 months, mean 41 months, median 37 months. The GDD implantation surgeries were performed by 10 different glaucoma fellowship-trained surgeons and there was no significant association between individual surgeon and likelihood of GDD exposure $(p=0.202)$. In univariable analyses of potential explanatory variables including type of GDD, type of patch graft, total number of GDD in the eye, and location of GDD, only female gender $(p=0.011)$ and white race $(p=0.044)$ were associated with likelihood of exposure (table 1). Women having undergone GDD surgery had twice the odds of experiencing exposure than men who underwent GDD surgery (OR 2.00 (95\% CI 1.17 to 3.43)). We considered that the association between gender and exposure might be related to age, as the women in the sample were, on average, older at the time of surgery than the men (mean age of women 66 years, mean age of men 61 years, $\mathrm{p}=0.001)$. The test of interaction between age and gender was significant $(p=0.025)$, implying that age influenced the association between gender and exposure differently for women compared to men. As such, separate analyses were performed for men and women with regard to the association between age and exposure, revealing that increasing age was associated with increased likelihood of GDD exposure for men $(p=0.038)$, but not for women $(p=0.394)$.

\section{Multivariable analysis}

The interaction between age and race was not significant $(\mathrm{p}=0.109)$; as such, age, race and gender were considered together as explanatory factors for the outcome of exposure. In this multivariable logistic regression model, age was not associated with exposure $(\mathrm{p}=0.657)$; white race $(\mathrm{p}=0.046)$ and female gender $(\mathrm{p}=0.013)$ remained significantly associated with likelihood of exposure. The odds of a white female experiencing exposure of the GDD were 3.88 times that of a black male experiencing exposure.

\section{Survival analysis}

To account for duration of follow-up, the relationship between potential explanatory variables and the 
Table 1 Characteristics of participants and association with glaucoma drainage device exposure by univariable analysis

\begin{tabular}{|c|c|c|c|c|}
\hline Participant characteristics $(n=1073)$ & Total number (\%) & $\begin{array}{l}\text { Number with } \\
\text { exposure (\%) }\end{array}$ & p Value & OR $(95 \% \mathrm{Cl})$ \\
\hline \multicolumn{5}{|l|}{ Gender } \\
\hline Male & $483(45)$ & $20(4)$ & \multirow[t]{2}{*}{0.011} & \multirow[t]{2}{*}{$2.00(1.17$ to 3.43$)$} \\
\hline Female & $590(55)$ & $47(8)$ & & \\
\hline \multicolumn{5}{|l|}{ Race } \\
\hline White & $550(51)$ & $43(8)$ & \multirow[t]{3}{*}{$0.044^{*}$} & \multirow[t]{3}{*}{$1.69(1.01$ to 2.83$)$} \\
\hline Black & $503(47)$ & $24(5)$ & & \\
\hline Other & $20(2)$ & 0 & & \\
\hline \multicolumn{5}{|l|}{ Diabetes } \\
\hline Present & $300(28)$ & $23(34)$ & \multirow[t]{3}{*}{0.296} & \multirow[t]{3}{*}{$0.76(0.45$ to 1.28$)$} \\
\hline Not present & $711(66)$ & $42(63)$ & & \\
\hline Unknown & $62(6)$ & $2(3)$ & & \\
\hline \multicolumn{5}{|l|}{ Uveitis } \\
\hline History of uveitis & $209(20)$ & 8 (12) & \multirow[t]{3}{*}{0.410} & \multirow[t]{3}{*}{$0.78(0.44$ to 1.40$)$} \\
\hline No history of uveitis & $649(60)$ & $47(70)$ & & \\
\hline Unknown & $215(20)$ & $12(18)$ & & \\
\hline \multicolumn{5}{|l|}{ Glaucoma drainage device } \\
\hline Ahmed & $598(56)$ & $43(7)$ & \multirow[t]{4}{*}{$0.203^{*}$} & \multirow[t]{4}{*}{$0.67(0.42$ to 1.16$)$} \\
\hline Baerveldt & $470(44)$ & $24(5)$ & & \\
\hline Molteno & $4(<1)$ & 0 & & \\
\hline Shocket & $1(<1)$ & 0 & & \\
\hline \multicolumn{5}{|l|}{ Patch graft material } \\
\hline Eye bank sclera & $591(55)$ & $43(7)$ & \multirow[t]{6}{*}{$0.174^{*}$} & \multirow[t]{6}{*}{$1.50(0.85$ to 2.65$)$} \\
\hline Tutoplast sclera & $363(34)$ & $18(5)$ & & \\
\hline Sclera NOS & $75(7)$ & $4(5)$ & & \\
\hline Single-layer Tutoplast pericardium & $39(4)$ & $2(5)$ & & \\
\hline Double-layer Tutoplast pericardium & $14(1)$ & 0 & & \\
\hline Unknown & $1(<1)$ & 0 & & \\
\hline \multicolumn{5}{|l|}{ Location } \\
\hline Superior & $991(92)$ & $62(6)$ & \multirow[t]{2}{*}{0.955} & \multirow[t]{2}{*}{$1.03(0.40$ to 2.63$)$} \\
\hline Inferior & $82(8)$ & $5(6)$ & & \\
\hline \multicolumn{5}{|l|}{$\begin{array}{l}\text { Total number of glaucoma drainage } \\
\text { devices in eye }\end{array}$} \\
\hline One & $817(76)$ & 49 & \multirow[t]{4}{*}{$0.125 \dagger$} & \multirow[t]{6}{*}{$0.65(0.37$ to 1.13$)$} \\
\hline Two & $175(16)$ & 16 & & \\
\hline Three & $24(2)$ & 2 & & \\
\hline Four & $1(<1)$ & 0 & & \\
\hline & Value & & $p$ Value & \\
\hline Age (years, mean $\pm S D ;$ median) & $64 \pm 16 ; 66$ & $65 \pm 16 ; 69$ & 0.335 & \\
\hline
\end{tabular}

outcome of GDD exposure was also queried by survival analysis. The mean time from GDD implant surgery to GDD exposure was $25 \pm 19$ months. The mean time to exposure for females was $23 \pm 18$ months and for males was $31 \pm 20$ months. Survival analysis revealed that females experienced exposure of the GDD earlier in the course of follow-up than men $(\mathrm{p}=0.024$, figure 1$)$. White patients experienced exposure earlier than African-American patients (0.026). Survival of the GDD without exposure was not associated with location of the GDD, history of multiple GDD, diabetes, uveitis, type of glaucoma or type of patch graft used $(p=0.239-0.669)$.

\section{DISCUSSION}

GDD surgery is becoming increasingly common, ${ }^{1}$ and is a valuable tool in the management of glaucoma. Exposure of the implant, however, is one of the more frequent $^{2-4} 6$ and challenging complications of GDD surgery. ${ }^{8}$ To better inform both surgeons and patients about the risks and benefits of GDD surgery, we need a clearer understanding of the risk factors associated with exposure of the implant.

Most of the studies which have provided evidence for rates of GDD exposure have included only one type of implant, such as the Baerveldt, ${ }^{6}$ or Ahmed. ${ }^{4}{ }^{10}$ One 


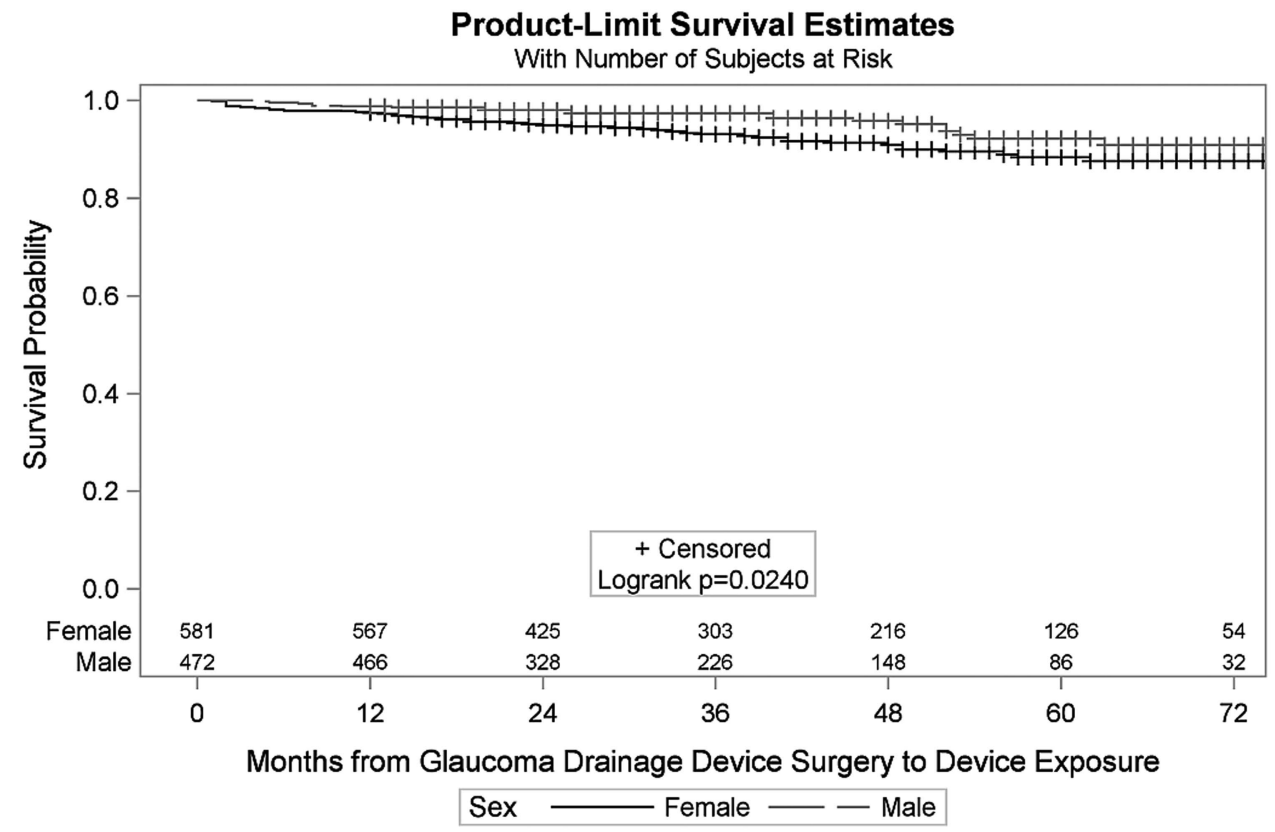

Figure 1 Survival probabilities for tube exposure for women and men.

study of exposure outcomes included both Baerveldt and Ahmed implants but focused primarily on outcome differences based on patch graft materials. ${ }^{11}$ Another recent study included multiple types of implants and did not find a difference in exposure rates. ${ }^{3}$ Our study included 598 (56\%) Ahmed and 470 (44\%) Baerveldt GDD implants. We did not find a difference in exposure outcomes related to type of GDD $43(7 \%)$ of the participants with Ahmed implants experienced exposure compared to $24(5 \%)$ of the participants with Baerveldt implants $(p=0.203)$. Exposure rates for both types of implants fell within the range of exposure rates previously published. ${ }^{3-7}$

Previous studies have compared the exposure-related outcomes for specific patch graft materials and found that single-layer pericardium is associated with greater risk of exposure than double-layer pericardium ${ }^{13}$ and pericardial patch grafts in general are associated with greater likelihood of exposure than corneal patch grafts. ${ }^{12}$ Some studies, however, have failed to find an association between patch graft material and exposure rates. ${ }^{3}{ }^{4}$ In our study, a variety of patch graft materials were employed primarily eye bank sclera $(n=591,55 \%)$, Tutoplast (IOP Ophthalmics, Costa Mesa, California, USA) sclera $(\mathrm{n}=363,34 \%)$ and to a lesser extent, single $(\mathrm{n}=39,4 \%)$ and double-layer $(\mathrm{n}=14,1 \%)$ Tutoplast pericardium. The numbers of participants receiving pericardial patch grafts were too small for adequate analysis, but there were no incidences of exposure in our double layer pericardial patch group as there were none in the 59 cases in Moster's study. ${ }^{13}$ We did not find an association between the likelihood of exposure for participants receiving eye bank sclera $(n=43$ exposure, $7 \%)$ compared to participants receiving Tutoplast sclera $(\mathrm{n}=18$ exposures, 5\%; $\mathrm{p}=0.174)$. Although failure to detect a difference does not mean that a differential likelihood of exposure does not exist, given the similar rates of exposure between participants with eye bank sclera and participants with Tutoplast sclera, a much larger sample would be needed to detect significant difference. For example, based on the rates of exposure in our study, we estimate that 2327 participants receiving eye bank and 2327 participants receiving Tutoplast sclera would be needed to have $90 \%$ power to detect a significant difference in likelihood of exposure, with $\alpha=0.05$.

Reports of GDD exposure related to location of the implant have varied. In a series of Ahmed GDD surgeries, implants placed in the inferior quadrants were more likely to expose than implants located superiorly. ${ }^{10}$ Another study of Ahmed implants, however, found higher rates of early wound dehiscence for GDD implants located inferiorly, but no association between location and later GDD exposure. ${ }^{11}$ We also did not find a difference in exposure for inferior $(\mathrm{n}=5$ exposures, $6 \%$ ) versus superior location of the device ( $\mathrm{n}=62$ exposures, $6 \%$; $\mathrm{p}=0.955$ ). Furthermore, we did not find a difference in the likelihood of exposure for GDD implants in eyes with a single implant $(\mathrm{n}=49$ exposures, $6 \%)$ compared to eyes with pre-existing GDD prior to the surgery included in the study ( $\mathrm{n}=18$ exposures, 9\%; $\mathrm{p}=0.125$ ). We purposely, however, only considered the first GDD surgery within the study period for each participant, so it is possible that we underestimated the rates of exposure for participant with multiple implants by excluding subsequent GDD surgeries and exposure events in the same participant. We did not investigate concurrent ophthalmic surgery as a risk factor for GDD exposure, 
although this has recently been reported to be a significant factor associated with GDD exposure. ${ }^{3}$

We observed that women had twice the odds of experiencing exposure of the GDD compared to men. In attempts to understand this association, we considered that this finding may be confounded by age, as the women in the study population were, on average, older at the time of surgery than the men. Indeed, the test of interaction between age and gender with regard to likelihood of GDD was significant, indicating that age influences the likelihood of exposure differently for men and women. For men, increasing age inferred a greater likelihood of exposure; multivariable analysis revealed that women, however, were more likely to experience exposure of the GDD than men regardless of age. Moreover, in survival analysis, women experienced GDD exposure earlier in the course of follow-up than men. A genderrelated difference in GDD exposure is a new finding: in a study of 11 cases of GDD exposure and 44 participants with GDD and without exposure, age and gender were not related to likelihood of exposure of the device. ${ }^{4}$ Likewise, in a recent study of 339 eyes of 332 participants with GDD surgery including 28 eyes with GDD exposure, gender was not associated with likelihood of exposure. ${ }^{3}$ Our study, however, defined exposure as occurring at least 1 month postoperatively and it is possible that female gender is a more important risk factor for later exposure than in early would dehiscence. The number of participants included in our study is also larger, improving our power to detect outcome differences.

We found that white race (compared to black race) was associated with greater likelihood of GDD exposure, although the association was not as strong (OR 1.69 (95\% CI 1.01 to 2.83)). When considered together in a multivariable model, white race $(p=0.046)$ and female gender $(p=0.013)$ remained significantly associated with GDD exposure. In a recent case-control study of GDD exposures, Koval et $a l^{14}$ found Hispanic race (vs non-Hispanic ethnicity including black and white participants), to be a risk factor for exposure. Our study population did not include enough persons of Hispanic descent for analysis. Koval et al matched controls to cases based on gender, so the influence of gender on exposure risk as not explored. Although, to our knowledge, no previous studies have found black race to be a risk factor for primary exposure of the GDD, interestingly, black race was a risk factor for requiring multiple surgeries following repair of GDD exposure in a series of exposure cases. ${ }^{9}$ The same study also found diabetes to be a risk factor for failure of primary revision of an exposed GDD. We did not find an association between diabetes and GDD exposure. Presence or absence of diabetes was determined by review of the problem list in our study, rather than laboratory tests and some participants with diabetes may have been classified as participants with no diabetes. Consistent with our findings, however, investigators in Korea did not find an association between diabetes and GDD exposure. ${ }^{4}$
We considered potential factors that might explain the increased likelihood of GDD exposure in women. Friction of the implant against the ocular tissues may contribute to late exposure of the GDD. In general, the orbital dimensions of women are smaller than men, with lower average height of the orbit and width of the orbital fissure for women compared to men. ${ }^{16}$ Rates of GDD exposure are higher in children than in adults, supporting the theory that a 'tight' orbit is associated with increased likelihood of exposure. ${ }^{17} \mathrm{~A}$ mechanically tighter orbit may contribute to the racial differences in exposure outcomes noted in our study. On average, the palpebral fissure width is greater for blacks than for whites. $^{18}$

Repetitive microtrauma may compromise conjunctival integrity, increasing the risk for exposure. Ocular dryness may exacerbate friction between the eyelids and ocular surface. The median age at surgery for the participants in our study was 66 years, suggesting that many women were postmenopausal and may have hormone-related dry eye syndrome. A prospective study quantifying dry eye in patients undergoing GDD surgery may help determine if dry eye influences the association between female gender and GDD exposure.

Our study has several limitations. Some patients may have experienced GDD exposure more than 1 year after surgery and have been treated elsewhere. Our dataset was limited to the history in the medical record and documentation may not always be complete. For example, we did not find an association between uveitis and exposure. The history of uveitis may not have been recorded for every patient, however, in children, uveitis is a risk factor for GDD exposure. ${ }^{17}$ Likewise, we could not examine the contribution of dry eye disease. Similarly, we limited our surgical history to previous GDD surgery; other prior conjunctival surgeries may be associated with GDD exposure, but was not uniformly recorded for each participant, especially participants who may have had surgeries prior to treatment at our institution.

We purposefully limited our study to cases of exposure occurring more than 1 month from surgery to exclude operative wound dehiscence; accordingly, the findings should not be extrapolated to include early exposures. Indeed, other studies have found alternative risk factors for early wound dehiscence. ${ }^{11}$ We chose to exclude repeat surgeries in the same patient in order to not overrepresent patient-specific characteristics which might predispose an individual to exposure of the GDD. As such, the finding that women are at increased risk for exposure is even more robust.

To the best of our knowledge, this is the largest series of GDD surgeries reported which includes a variety of types of GDD implants and patch graft materials. As GDD surgeries become an increasingly common event in the management of glaucoma, further study is needed to understand why women are at greater risk of GDD exposure and what can be done to mitigate this risk. 
Contributors The study was conceptualised and designed by KWM, MMW, AK and HT. AL performed chart abstractions. SS performed statistical analyses KWM drafted the manuscript. All authors were involved in critical revision of the manuscript.

Funding This research received no specific grant from any funding agency in the public, commercial or not-for-profit sectors.

Competing interests Dr Muir receives salary support from a VA Health Services Research \& Development Career Development Award.

Ethics approval Duke IRB.

Provenance and peer review Not commissioned; externally peer reviewed.

Data sharing statement The authors are happy to answer any questions about the data. Any protected health information analysed in this study belongs to Duke and cannot be shared outside of the institution.

Open Access This is an Open Access article distributed in accordance with the Creative Commons Attribution Non Commercial (CC BY-NC 3.0) license, which permits others to distribute, remix, adapt, build upon this work noncommercially, and license their derivative works on different terms, provided the original work is properly cited and the use is non-commercial. See: http:// creativecommons.org/licenses/by-nc/3.0/

\section{REFERENCES}

1. Ramulu PY, Corcoran KJ, Corcoran SL, et al. Utilization of various glaucoma surgeries and procedures in Medicare beneficiaries from 1995 to 2004. Ophthalmol 2007;114:2265-70.

2. Gedde SJ, Schiffman JC, Feuer WJ, et al. Treatment outcomes in the Tube Versus Trabeculectomy (TVT) study after five years of follow-up. Am J Ophthalmol 2012;153:789-803.

3. Trubnik V, Zangalli C, Moster MR, et al. Evaluation of risk factors for glaucoma drainage device-related erosions: a retrospective case-control study. J Glaucoma [Epub ahead of print Dec 10 2013].

4. Byun YS, Lee NY, Park CK. Risk factors of implant exposure outside the conjunctiva after Ahmed glaucoma valve implantation. Japan Ophthalmol 2009;53:114-19.
5. Smith MF, Doyle JW, Ticrney JW Jr. A comparison of glaucoma drainage implant tube coverage. J Glaucoma 2002;11:143-7.

6. Krishna R, Godfrey DG, Budenz DL, et al. Intermediate-term outcomes of 350-mm(2) Baerveldt glaucoma implants. Ophthalmology 2001;108:621-6.

7. Gedde SJ, Herndon LW, Brandt JD, et al. Postoperative complications in the Tube Versus Trabeculectomy (TVT) study during five years of follow-up. Am J Ophthalmol 2012;153:804-14.

8. Gedde SJ, Scott IU, Tabandeh $\mathrm{H}$, et al. Late endophthalmitis associated with glaucoma drainage implants. Ophthalmology 2001;108:1323-7.

9. Huddleston SM, Feldman RM, Budenz DL, et al. Aqueous shunt exposure: a retrospective review of repair outcome. J Glaucoma 2013;22:433-8.

10. Pakravan M, Yazdani S, Shahabi C, et al. Superior versus inferior Ahmed glaucoma valve implantation. Ophthalmology 2009;116:208-13.

11. Geffen N, Buys YM, Smith M, et al. Conjunctival complications related to Ahmed glaucoma valve insertion. J Glaucoma 2014;23:109-14.

12. Wigton E, Swanner CJ, Joiner W, et al. Outcomes of shunt tube coverage with glycerol preserved cornea versus pericardium. J Glaucoma 2014;23:258-61.

13. Lankaranian D, Reis R, Henderer JD, et al. Comparison of single thickness and double thickness processed pericardium patch graft in glaucoma drainage device surgery: a single surgeon comparison of outcome. J Glaucoma 2008;17:48-51.

14. Koval MS, El Sayyad FF, Bell NP, et al. Risk factors for tube shunt exposure: a matched case-control study. J Ophthalmol 2013;2013:196215

15. Horvath MM, Winfield S, Evans S, et al. The DEDUCE guided query tool: providing simplified access to clinical data for research and quality improvement. J Biomed Inform 2011;44:266-76.

16. Ferrario VF, Sforza C, Colombo A, et al. Morphometry of the orbital region: a soft-tissue study from adolescence to mid-adulthood. Plast Reconstr Surg 2001;108:285-92; discussion 93.

17. Morad Y, Donaldson CE, Kim YM, et al. The Ahmed drainage implant in the treatment of pediatric glaucoma. Am J Ophthalmol 2003;135:821-9.

18. Price KM, Gupta PK, Woodward JA, et al. Eyebrow and eyelid dimensions: an anthropometric analysis of African Americans and Caucasians. Plast Reconstr Surg 2009;124:615-23. 